\title{
EFFECT OF STRESS PATH, SIZE AND SHAPE ON THE OPTIMUM PARAMETERS OF A BRITTLE-DUCTILE CONCRETE MODEL
}

\author{
Mojtaba LABIBZADEH, Reza HAMIDI \\ Department of Civil Engineering, Faculty of Engineering, Shahid Chamran University of Ahvaz, Ahvaz, Iran
}

Received 06 April 2017; accepted 05 December 2017

\begin{abstract}
The main purpose of this study is to investigate the effects of the stress path, size and shape of the specimen, on the optimum values of the plastic input parameters of Concrete Damaged PlasticityCDP- model of the ABAQUS software. This study concentrates on eccentricity, dilatation angle and viscosity input parameters. To finding optimum values of the mentioned parameters, an objective function which estimates the error of the model predictions was defined and tried to minimize. For evaluating the effect of the stress path on this optimization procedure, four different standard tests were simulated by the ABAQUS software: uniaxial compression, dual compression, dual-border cut specimen and three-point bending tests. For studying the effect of the size of the specimen, the three-point bending test, with three different sizes of the beam were modeled in the ABAQUS software. Furthermore, for investigating the effect of the specimen shape on the input parameters, a notched disk in a tensile stress field was modeled. Obtained results demonstrated that the path of the stress can influence significantly on the optimum values of the input parameters, while it was revealed that the CDP model can automatically take into account the size and shape effects in the simulation.
\end{abstract}

Keywords: concrete damage plasticity, stress path, size effect, shape effect, ABAQUS.

\section{Introduction}

Accurate modeling of the behavior of concrete structures under the action of different loading conditions is a crucial task. To this end, an enhanced material model of concrete is needed to be formulated and placed into a powerful finite element program. This concrete material model should inevitably be capable of appropriately representing both elastic and plastic behavior of the concrete in compression and tension. All other stress fields which develop in a problem domain can be captured by proper combinations of these two basic behaviors. Furthermore, such a material model also has to be able to preserve its efficiency if the geometrical characteristics of the problem domain are changed. In other words, a powerful material model must be so formulated to become insensitive to the size and the shape of the specimen. These two mentioned attributes are essential for every material model to become a general applicable model. The Concrete Damaged Plasticity or in the abbreviated form as CDP model of the ABAQUS standard software is a sophisticated material model of concrete and the authors of this paper are intended to investigate whether this model satisfies the two above mentioned fundamental conditions.

The two main typical concrete failure mechanisms are cracking due to tension and crushing due to compression. CDP model is a continuum, plasticitydamage based model for concrete (Dassault Systèmes 2008). It considers the two mentioned above concrete failure mechanisms. The CDP model of the ABAQUS software has been developed based on a theory first introduced by Lubliner et al. (1989) and was developed later by Lee and Fenves (1998). Much research has 
been carried out in recent years on CDP model among them the followings are the most cited ones. Birtel and Mark (2006) have conducted a parametric study on the shear failure mechanism of a reinforced concrete beam using CDP for concrete constitutive model. Sadeghi and Fathali (2007) investigated the degradation of old concrete bridges under unexpected loads using CDP model. Kmiecik and Kaminski (2011) have Modeled reinforced and composite concrete structures with concrete strength degradation and discussed the input parameters that it used by the CDP model. Sadeghi and Fathali (2012) analyzed concrete deck of a bridge using CDP in grillage analogy (GA). Javier Rodrigues, Francisco Martinez, and Joaquin Marti (2013) have been developed a methodology for mechanical behavior simulation of the concrete material based on the CDP model and implemented the data from the triaxial tests at various confining pressures for calibrating the dilation angle input parameter. Szczecina and Winnicki (2015) have conducted a sensitivity analysis of the CDP model input parameters in ABAQUS and focused on the viscosity parameter in tension test and dilation angle in compression test. Yusuf Sümer and Muharrem Aktaş (2015) have defined input parameters for CDP model by simulating three different four-point bending reinforced beams with different grades of concrete and other specifications. Labibzadeh (2015) and Labibzadeh and Elahifar (2015) have employed the CDP model for the numerical simulation of the strengthened RC slabs with CFRPs and calibrated the input parameters using some different tests such as biaxial compression test, three-point bending concrete beam test and four-point bending reinforced concrete beam test. Labibzadeh et al. (2017a) presented a new method for finding the optimum values for input parameters of the CDP model which can guarantee the uniqueness and the precise of the obtained results. They suggested the use of the results of the different standard tests of the concrete for the calibrating the input parameters of the CDP model. Labibzadeh et al. (2017b) implemented a modified version of CDP model in an innovative inverse method to evaluate the long-term behavior of an aged concrete structure. Sadeghi et al. (2017) conducted a research on the mechanism of transfer of load from railway to concrete slab support and improved the current practice of design of such slabs using CDP with a nonlinear model.

Authors of this present paper decided to find a set of optimum plastic parameters of the CDP model considering two important effective factors which have not been considered in the above mentioned studies: stress path and size effects. To take into account the effects of the first factor, four standard tests, i.e., uniaxial compression, biaxial compression, dual-border cut specimen and three-point bending tests have been selected form validated literature and simulated by the ABAQUS software. The concrete characteristics used in these three tests are very close to each other, therefore, this condition can provide a situation in which only the stress path plays the key role in obtaining different results. The procedure for study the effect of stress path on the input parameters of the CDP model was to define an objective function for each test and then to minimize it for each parameter by assuming the constant value for the other parameters. Hence, the effect of each parameter can be checked in the result of finite element analysis (FEA). The objective or residual function, $\mathrm{R}$, is considered as the root of the ratio of the sum of the squares of the differences between the value of stresses obtained from the experiment and FEA results in the same strain to the sum of the squares of the experimental data in the case of uniaxial and biaxial compression tests. For the other two remained tests; dual-border cut specimen and three point bending, the objective function is defined as the root of the ratio of the sum of the squares of the differences between the value of loads obtained from the experiment and FEA results in the same displacement to the sum of the squares of the experimental data. For assessment of the size effect on the other hand, the three-point bending test with two extra sizes have been modeled named as medium and large sizes and the same tensile strength of the concrete $\left(f_{t}\right)$ were used for these three sized three-point bending tests. For evaluating the effect of shape of the input parameters of the CDP model, a notched disk with mode I cracking pattern of failure was modeled in ABAQUS software. For this test, the optimum input parameters obtained for threepoint bending test were utilized.

\section{Compressive and tensile behavior of the CDP model}

The constitutive model used in this study for concrete is the Concrete Damaged Plasticity (CDP) Model formulated in the ABAQUS software. It assumes that the main two failure mechanisms are tensile cracking and compressive crushing of the concrete material. The evolution of the yield (or failure) surface is controlled 
by two hardening variables, $\tilde{\varepsilon}_{t}^{p l}$ and $\tilde{\varepsilon}_{c}^{p l}$ (tensile and compressive equivalent plastic strains, respectively) related to failure mechanisms under tensile and compressive loading, respectively. The model assumes that the uniaxial tensile and compressive response of concrete is characterized by a combined damage plasticity phenomenon.

According to these figures, under uniaxial tension, the stress-strain response follows a linear elastic relationship until the value of the fracture stress, $\sigma_{t o}$, is reached. This cracking stress corresponds to the onset of visible cracking in the concrete material. Beyond this stress, the growth of cracks is represented macroscopically with a softening stress-strain response, which induces strain localization in the concrete structure. When the concrete specimen is unloaded from any point on the strain softening branch of the stressstrain curve, the unloading response is weakened and the elastic stiffness of the material is degraded or damaged. The degradation of the elastic stiffness is characterized by a damage variable in tension, $d_{t}$, which can take values from zero, representing the undamaged material, to one, representing the total loss of strength. Under uniaxial compression, the response is linear elastic until the value of the initial yield, $\sigma_{c o}$ is met. In the plastic regime, the response is typically characterized by strain hardening followed by strain softening beyond the ultimate stress, $\sigma_{c u}$. When the concrete specimen is unloaded after the initial elastic behavior, the unloading response is weakened and the elastic stiffness of the material is reduced or damaged. The degradation of the elastic stiffness is characterized by a damage variable, $d_{c}$,which can take values from zero, representing the undamaged material, to one, representing the total loss of strength. It is assumed that the uniaxial stress-strain curves can be converted into stress versus plastic-strain curves using the Eq. (1) and (2):

$$
\begin{aligned}
& \tilde{\varepsilon}_{t}^{p l}=\tilde{\varepsilon}_{t}^{c k}-\frac{d_{t}}{\left(1-d_{t}\right)} \frac{\sigma_{t}}{E_{0}} ; \\
& \tilde{\varepsilon}_{c}^{p l}=\tilde{\varepsilon}_{c}^{i n}-\frac{d_{c}}{\left(1-d_{c}\right)} \frac{\sigma_{c}}{E_{0}},
\end{aligned}
$$

where $\tilde{\varepsilon}_{t}^{p l}$ and $\tilde{\varepsilon}_{c}^{p l}$ are equivalent plastic strains in tension and compression respectively, the $\tilde{\varepsilon}_{t}^{c k}$ is the crack strain, $\tilde{\varepsilon}_{c}^{i n}$ is the inelastic strain, $d_{t}$ and $d_{c}$ are the damage variables in tension and compression respectively, $\sigma_{t}$ and $\sigma_{c}$ are the tension and compression stresses respectively and finally $E_{0}$ is the initial undamaged elastic stiffness of the material.
In this study, to simulate the concrete behavior in compression for the simulation of the two first tests, i.e., the uniaxial and biaxial compression tests, the experimental results of Kupfer and Gerstle have been used (Kupfer, Gerstle 1973). But, for the third test, i.e., three-point bending test, the compressive stress-strain relationship of concrete model proposed by Popovics was incorporated (Popovics 1973). In this model, the value of compressive concrete stress $\left(f_{c}\right)$ is a function of concrete strain $\left(\varepsilon_{c}\right)$ which can be derived from the following equation:

$$
\frac{f_{c}}{f_{c}^{\prime}}=\frac{n\left(\frac{\varepsilon_{c}}{\varepsilon_{0}}\right)}{n-1+\left(\frac{\varepsilon_{c}}{\varepsilon_{0}}\right)^{n k}},
$$

where $f_{c}^{\prime}$ is the ultimate compressive strength of concrete, $n$ is a curve-fitting factor (can be taken as $n=0.8+f_{c} / 17$ or $\left.n=\frac{E_{c}}{\left(E_{c}-E_{c}^{\prime}\right)}\right), E_{c}$ is the initial tangent modulus (can be taken as $E_{c}=6900+3300 \sqrt{f_{c}^{\prime}}$ ) $\varepsilon_{0}$ denotes the strain when $f_{c}$ reaches to $f_{c}^{\prime}$; $\varepsilon_{0}=\frac{f_{c}^{\prime}}{E_{c}}\left(\frac{n}{n-1}\right), E_{c}^{\prime}$ is the tangent modulus of concrete at $f_{c}^{\prime}, E_{c}^{\prime}=\frac{f_{c}^{\prime}}{\varepsilon_{0}}$, and $k$ is a factor to control the slope of the stress-strain curve:

$$
\begin{aligned}
& \text { If }\left(\frac{\varepsilon_{c}}{\varepsilon_{0}}\right) \leq 1.0 \rightarrow k=1.0 ; \\
& \text { if }\left(\frac{\varepsilon_{c}}{\varepsilon_{0}}\right)>1.0 \rightarrow k=0.67+\frac{f_{c}^{\prime}}{62} \geq 1.0 .
\end{aligned}
$$

Many researchers proposed a stress-strain relationship for concrete in tension. The strain-softening part (after cracking) of the model can be linear, bilinear or reciprocal function (Wang, Hus 2001; Shima et al. 1987). In this study, for the concrete a bi-linear strain-softening model were incorporated to simulate the concrete behavior in tension (Massicotte et al. 1990). The bi-linear strain-softening model decreases from point $\left(\varepsilon_{c r} ; f_{t}^{\prime}\right)$ to point $\left(5 \varepsilon_{c r} ; f_{t}^{\prime} / 3\right)$ with the slop of $\left(f_{t}^{\prime} / 6 \varepsilon_{c r}\right)$ and from point $\left(5 \varepsilon_{c r} ; f_{t}^{\prime} / 3\right)$ to $\left(16 \varepsilon_{c r} ; 0\right)$ with the slop of $\left(f_{t}^{\prime} / 33 \varepsilon_{c r}\right)$.

\section{Plastic input parameters of the CDP model}

To define the CDP model in the software, three main groups of the input parameters including of plasticity, compressive behavior and tensile behavior must be entered in the package by the user. To complete the 
compressive and tensile behavior parts, the experimental results of the uniaxial compression and tension tests are utilized respectively. However, the plasticity input parameters includes five different parameters as dilation angle $\psi$ in the p-q plane, flow potential eccentricity $(\varepsilon)$, the ratio $f_{b o} / f_{c o}$ of biaxial compressive yield stress to uniaxial compressive yield stress, the coefficient $K_{c}$ of the second stress invariant on the tensile meridian to that on the compressive meridian for the yield function and viscosity parameter. Three parameters of the mentioned parameters including dilation angle $(\psi)$, eccentricity $(\varepsilon)$ and viscosity parameter have been calibrated in this study while for two the other remained parameters the default values have been used. Value of the $f_{b o} / f_{c o}$ according to the Kupfer and Gerstle (1973) considered as 1.16 and the value of $K_{c}$ is defined as 0.67 for concrete (Dassault Systèmes 2008).

\section{Effect of stress paths on the input parameters}

\subsection{Compression tests}

The first two tests for calibration of the input parameters were selected as uniaxial and biaxial compression tests. These two tests were selected as representatives for stress paths lying on the compressive and tensile meridians in the $\mathrm{p}-\mathrm{q}$ coordinate system respectively. $\mathrm{P}$ and $\mathrm{q}$ are the equivalent stresses denote the hydrostatic and Von Mises stresses respectively. The dimensions of the cubic specimen were considered as $20 \mathrm{~cm}^{\star} 20 \mathrm{~cm}^{\star} 5 \mathrm{~cm}$. The size of the specimens was similar to the corresponding experimental samples implemented by Kupfer and Gerstle in their tests.

\subsubsection{Finite element model}

The finite element models of the cubic samples were simulated in ABAQUS software for the uniaxial and dual compression tests as shown in the Figures 1 and 2 respectively. For these FE models, the 8-noded linear hexahedral element (C3D8R) with a reduced integration formulation and a size element of $10 \mathrm{~mm}$ were chosen which gave the closest results when compared to the experimental results. This element size was selected after performing several sensitivity analyses with different element sizes and finding the best agreement with experimental data. For the uniaxial compression model, two rigid plates at the top and bottom of the cube and for the biaxial compression model two rigid plates at the bottom and lateral side of the cube were considered to simulate uniform pressure on the sur-

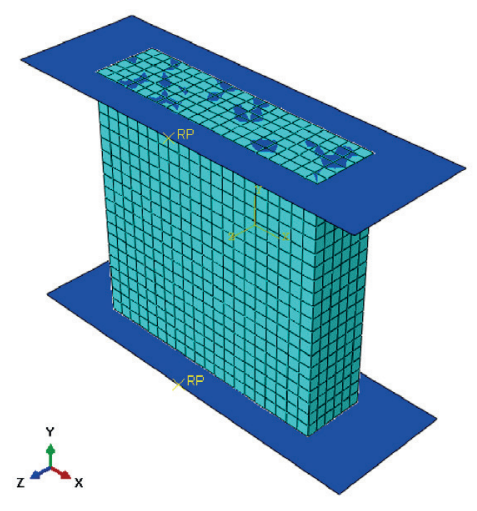

Fig. 1. Finite element model of the uniaxial compression test

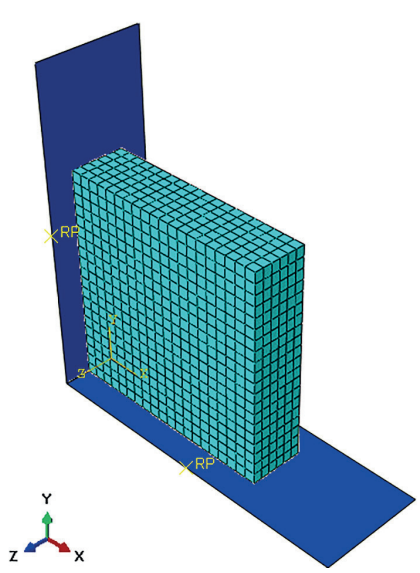

Fig. 2. Finite element model of the dual compression test

faces and avoiding the concentrated stress effects. The analysis was performed using the static-general step type of the analysis module after the initial step. The load was exerted on the cube surface by defining a reference point at the top plate and assigning a prescribed displacement to that reference point for the uniaxial compression model, and specifying a controlled displacement to the free top and lateral surfaces of the cube with a ratio of $1: 1$ for the biaxial compression test.

\subsubsection{Calibration of parameters}

The variation of the objective function, $\mathrm{R}$, defined earlier in the introduction section, versus different eccentricities with a dilation angle as $\psi=30$ and a viscosity parameter equal as 0.0001 for uniaxial and biaxial compression tests were illustrated in Figure 3 and figure 4 respectively. As it can be seen from these pictures, the changing in the eccentricity has a very low effect on the value of the objective function. The value of the objective function becomes smallest when the eccentricity is 0.1 . 


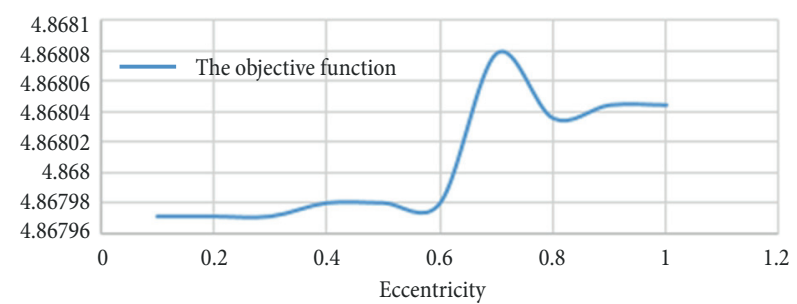

Fig. 3. The values of the objective function versus different eccentricities in uniaxial compression test

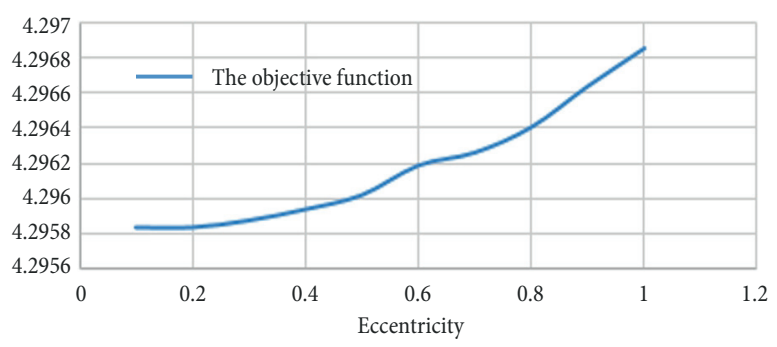

Fig. 4. The values of the objective function versus different eccentricities in biaxial compression test

Another parameter that must be optimized is the dilation angle. To this end, all other parameters assumed constant. The change of the objective function versus different dilation angles with eccentricity equal to 0.1 and viscosity parameter as 0.0001 for uniaxial compression and biaxial compression tests were depicted in Figures 5 and 6 respectively. As shown in these figures, the value of the dilation angle, $\psi=5$, minimizes the objective function, $\mathrm{R}$, but like the eccentricity this parameter has a low effect on the value of the objective function.

The last parameter which has been calibrated in this stage of the study is the viscosity parameter. Obtained results show that changing in the value of the viscosity parameter has more effect on the value of the objective function with respect to the other parameters. The trend of variation of the objective function versus different viscosity parameters with a dilation angle $\psi=30^{\circ}$ and eccentricity $\epsilon=0.1$ for uniaxial and biaxial compression tests was shown in Figures 7 and 8 respectively. As shown in these figures, the value of the objective function becomes minimum when the viscosity parameter reaches to 0.0001 .

The obtained optimized dilation angle $(\psi)$, eccentricity $(\epsilon)$ and viscosity parameter using uniaxial and biaxial compression tests have been summarized in Table 1. It is interesting to note that for these two tests, the same optimum input parameters have been obtained.

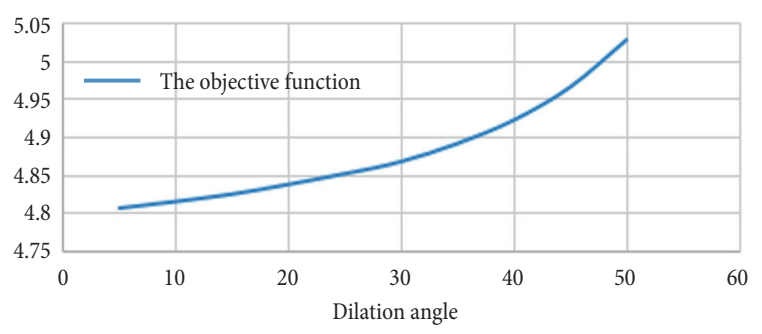

Fig. 5. The value of the objective function versus dilation angle in uniaxial compression test

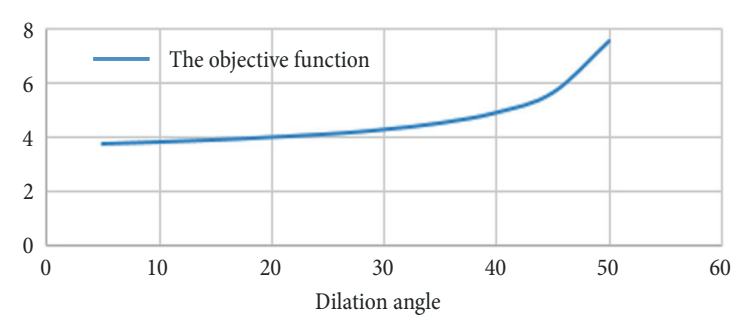

Fig. 6. The value of the objective function versus dilation angle in biaxial compression test

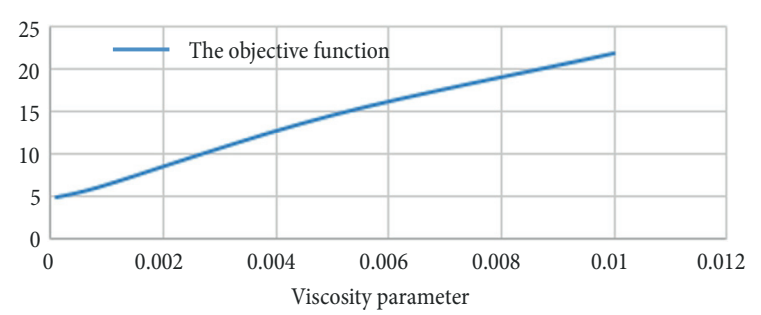

Fig. 7. The value of the objective function versus viscosity parameter in uniaxial compression test

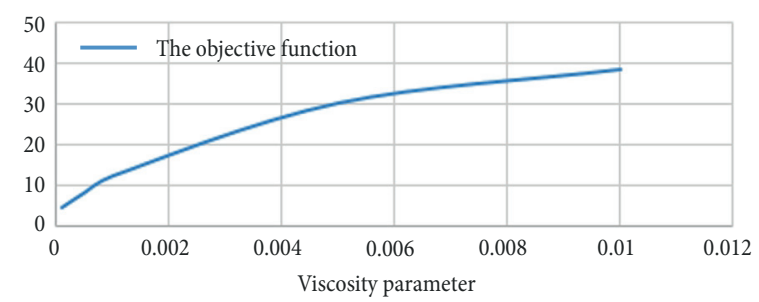

Fig. 8. The value of the objective function versus viscosity parameter in biaxial compression test

Table 1. Selected parameters which minimize the objective function in uniaxial and biaxial compression test

\begin{tabular}{|c|c|c|}
\hline number & parameter & value \\
\hline 1 & $\epsilon$ & 0.1 \\
\hline 2 & $\psi$ & 5 \\
\hline 3 & Viscosity parameter & 0.0001 \\
\hline
\end{tabular}

Comparisons between axial stress-strain curves of uniaxial and biaxial compression tests with the corresponding experimental curves of Kupfer and Gerstle (1973) were made and shown in Figure 9 and Figure 10 respectively using the obtained input parameters in 
Table 1. It can be seen from these figures that these parameters can simulate the behavior of concrete accurately and resulted in a good agreement between the finite element analysis (FEA) and the experiment. The percentages of error in estimating the peak stress in uniaxial and biaxial compression tests are $0.7 \%$ and $2 \%$ respectively.

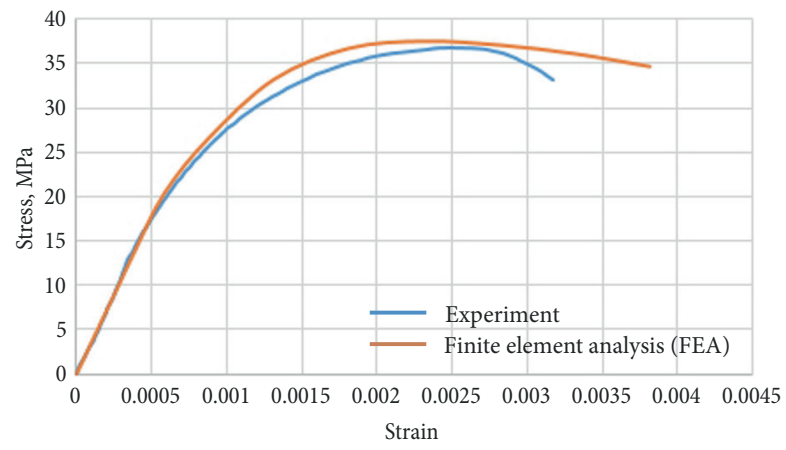

Fig. 9. Comparison of stress-strain responses of current study and experiment (Kupfer, Gerstle 1973) in uniaxial compression test

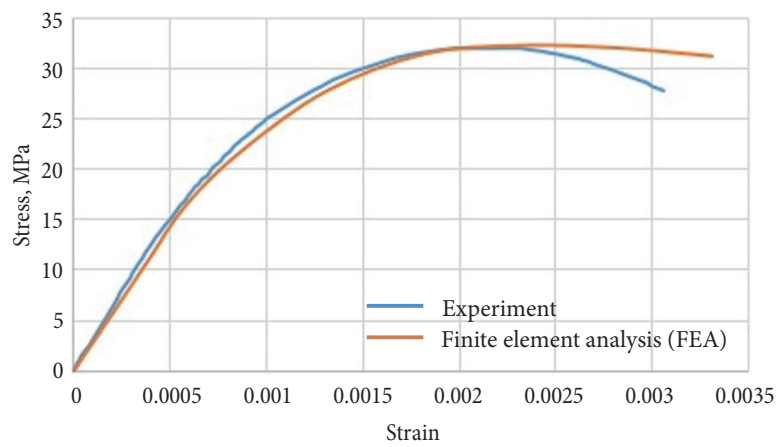

Fig. 10. Comparison of stress-strain responses of current study and experiment (Kupfer, Gerstle 1973) in biaxial compression test

\subsection{Dual Border-Cut Specimen test}

For considering the effect of shear stress path on the optimum input parameters of the CDP model, a dualborder cutted specimen was modeled in this study. Dimensions of this specimen are shown in Figure 11 ( $\mathrm{Zi}$ et al. 2007). In this test, at first, the specimen is loaded by shear force without any vertical force. At a distinct level of shear load, the pattern of loading is changed and vertical load is then applied.

The material properties used for developing model in ABAQUS software are according to (Zi et al. 2007) as compressive strength $\left(f_{c}^{\prime}=26 \mathrm{MPa}\right)$, splitting tensile strength $\left(f_{s}=3.78 \mathrm{MPa}\right)$, that yields according to the CEB-FIP model code a tensile strength of $\left(f_{t}=0.8 f_{s}\right)$, initial Young's Modulus $(E=29 \mathrm{GPa})$, Poisson's ratio $(\vartheta=0.22)$.

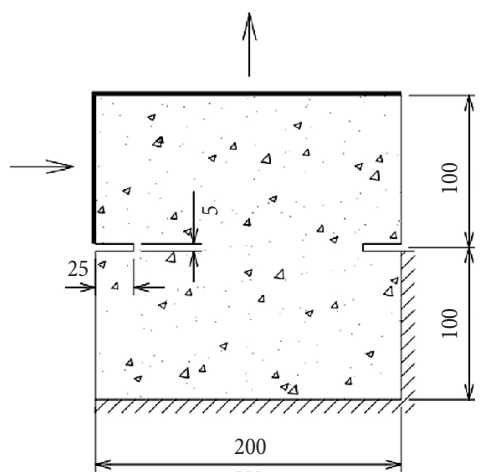

Fig. 11. A dual-border cut specimen (Zi et al. 2007)

\subsubsection{Finite element model}

The finite element model developed in the ABAQUS software is depicted in Figure 12. For modeling of the specimen, the most elements used are C3D8R solid element and near to the grooves the C3D4 tetrahedral element was used. The element size after doing several sensitivity analyses was considered equal to $10 \mathrm{~mm}$ for the whole of the specimen except for the upper and lower parts in which the $15 \mathrm{~mm}$ element size was utilized. The total number of elements used for modeling was obtained as 14583 .

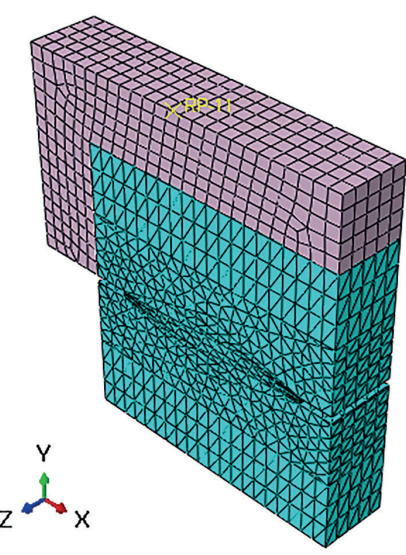

Fig. 12. Dual-border cut specimen under shear load

\subsubsection{Calibration of the parameters}

Figures 13 to 15 represent the variation of the objective function versus the eccentricity, dilation angle and viscosity parameters respectively. The optimum values for these input parameters have been summarized in $\mathrm{Ta}$ ble 2. The load-displacement curve of ABAQUS model developed in current study using the input parameters of Table 2 has been compared with the corresponding curve obtained from experiment ( $\mathrm{Zi}$ et al. 2007) in Figure 16. As it can be seen from this figure, there is a good agreement between these two curve types. 


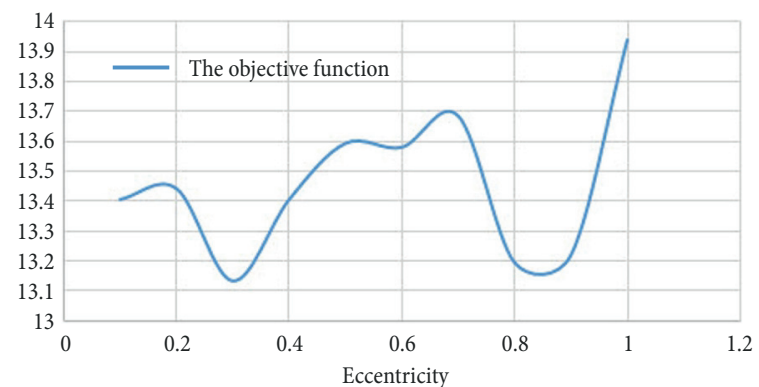

Fig. 13. The values of the objective function versus different eccentricities in dual-border cut test

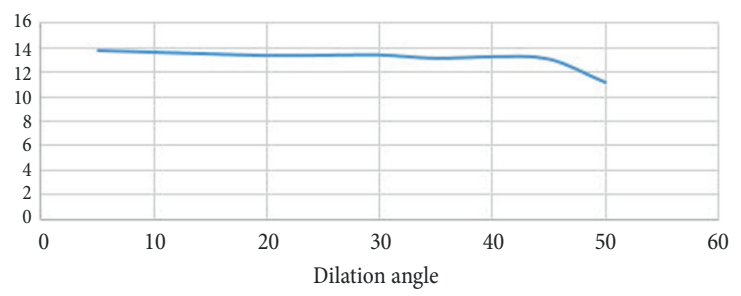

Fig. 14. The values of the objective function versus different dilation angles in dual-border cut test

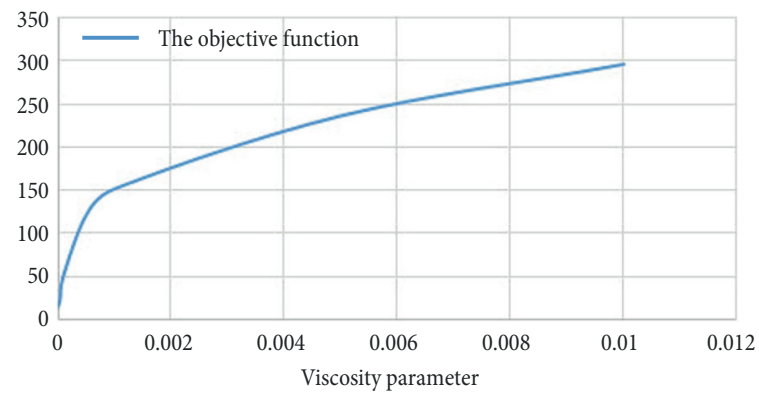

Fig. 15. The values of the objective function versus different viscosity parameters in dual-border cut test

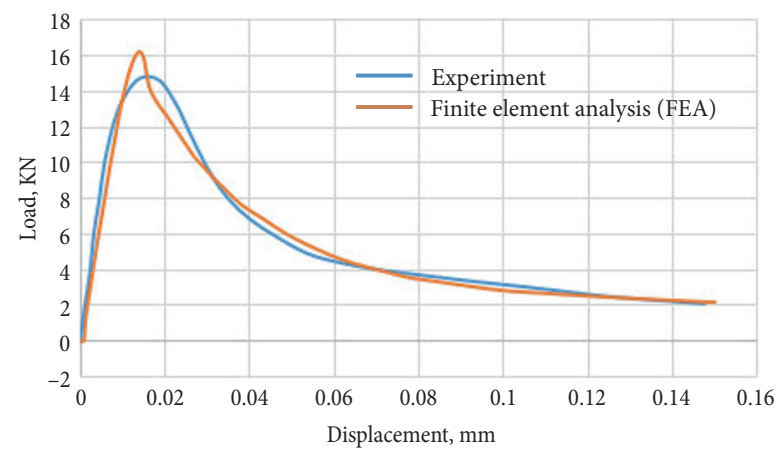

Fig. 16. Comparison of load-displacement responses obtained from FEA and experiment ( $\mathrm{Zi}$ et al. 2007) in three-point bending notched beam with small size

Table 2. Selected parameters which minimize the objective function in dual-border cut test

\begin{tabular}{|c|c|c|}
\hline number & parameter & value \\
\hline 1 & $\epsilon$ & 0.3 \\
\hline 2 & $\psi$ & 50 \\
\hline 3 & Viscosity parameter & 0.00001 \\
\hline
\end{tabular}

\subsection{Three-point bending of a Notched beam test}

A simply supported notched beam with a concrete tensile strength of $f_{t}=3.6 \mathrm{MPa}$ was selected as another example for assessment of the effect of stress path on the CDP input parameter performance. In this test, the mode I of tensile cracking has been simulated. The tensile strength of the concrete in this test is very close to the corresponding value in the previous two tests. The dimensions of the beam are illustrated in Figure 17, $h{ }^{\star} L=8 \mathrm{~cm} * 32 \mathrm{~cm}$ and the thickness of the beam is $4 \mathrm{~cm}$. The dimensions of the beam were chosen according to the corresponding beam which has been tested in laboratory by Le Bellego et al. (2003).

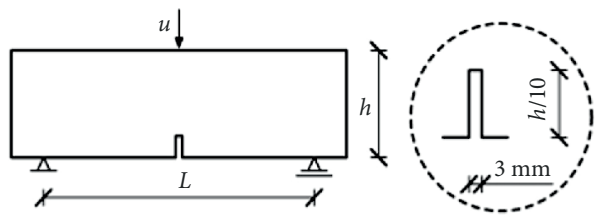

Fig. 17. Three-point notched beam specifications (Le Bellego et al. 2003)

\subsubsection{Finite element model}

Finite element analysis (FEA) was performed to develop reliable models based on experimental results which can simulate the behavior of three-point bending notched beam. As shown in the Figure 18, the FE model of three-point bending notched beam was developed using $A B A Q U S / C A E$. In the simulation, for the beam part, the linear tetrahedral element (C3D4) was used. The mesh size of the element in the middle of the beam was considered as $5 \mathrm{~mm}$ and in the remaining of the beam, the size of $10 \mathrm{~mm}$ was used which gave the best results in the simulation when they are compared to the experiment. The analysis was

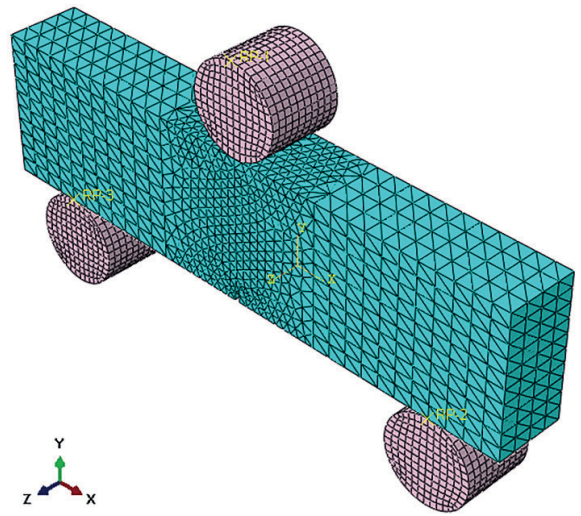

Fig. 18. Finite element model of the three-point bending beam with small size 
defined in static-general step. The loading was exerted on the beam by defining a reference point at the top of the beam and assigning a prescribed displacement to that reference point.

\subsubsection{Calibration of parameters}

The variation of the objective function, $\mathrm{R}$, versus different eccentricities with a dilation angle $\psi=20$ and a viscosity parameter equal to 0.0009 was shown in Figure 19. As shown in this figure, changing in the eccentricity has a low effect on the value of the objective function and when the eccentricity, $\epsilon=0.8$, the residual function, $\mathrm{R}$, becomes minimized.

Another parameter that has been calibrated in the present study for three-point bending test is the dilation angle, for doing that all the other parameters assumed constant. The change of the objective function versus different dilation angle with $\epsilon=0.5$ and viscosity parameter $=0.0009$ was depicted in Figure 20. As shown in this figure, the value of dilation angle, $\psi=$ 25 , can minimize the objective function.

The last parameter that has been considered for the optimization was the viscosity parameter and it was observed that changing of the value of the viscosity parameter has more effect on the value of objective function rather than to other parameters. Figure 21 shows the performance of the value of the objective function versus different viscosity parameters with $\psi=20$ and $\epsilon=0.5$. As shown in the Figure 21, the value of the objective function when the viscosity parameter is reached to 0.0006 becomes minimized.

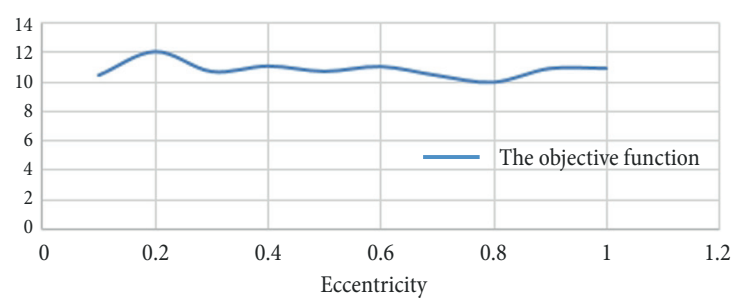

Fig. 19. The value of the objective function versus different eccentricities in three-point notched beam

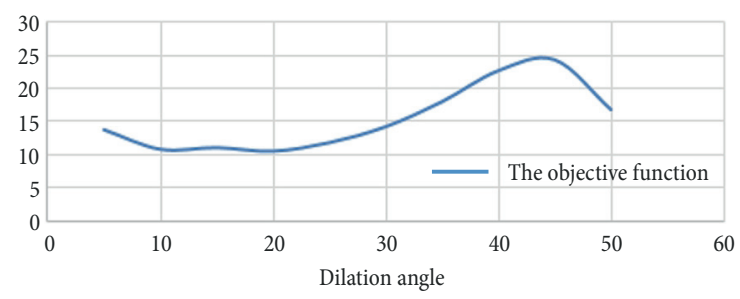

Fig. 20. The value of objective function versus dilation angle in three-point notch beam

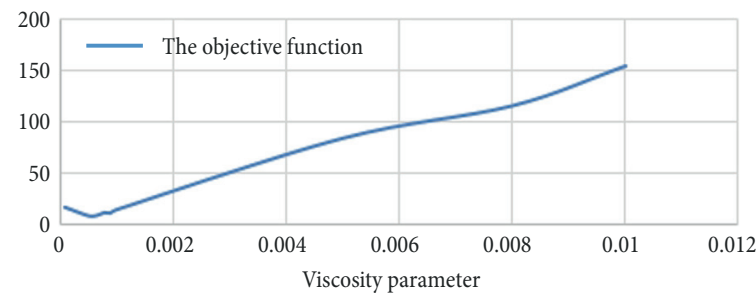

Fig. 21. The value of the objective function versus viscosity parameter in three-point notch beam

At the end of the optimization of input parameters by three-point bending test, three parameters: dilation angle $(\psi)$, eccentricity $(\epsilon)$ and viscosity parameter have been calibrated. Summary results for these selected parameters which minimize the value of the objective function were outlined in Table 3.

Table 3. Selected parameters which minimize the objective function in three-point notch beam

\begin{tabular}{|c|c|c|}
\hline number & parameter & value \\
\hline 1 & $\epsilon$ & 0.8 \\
\hline 2 & $\psi$ & 25 \\
\hline 3 & Viscosity parameter & 0.0006 \\
\hline
\end{tabular}

The optimum input parameters obtained in $\mathrm{Ta}$ ble 3 used for simulation the concrete behavior in the finite element model of the three-point bending test. The load-displacement curves obtained from experiment (Le Bellego et al. 2003) and finite element analysis (FEA) of the beam were compared in Figure 22. As shown in this figure, the result of the numerical simulation and experiment has a good agreement. The percentage of error in evaluation of peak load from $\mathrm{FE}$ analysis is about $6.3 \%$ when it is compared with its corresponding value obtained from test. Moreover, the peak of the curve in the FEA located in a place that is very close to the point that peak of the curve in the experiment is reached.

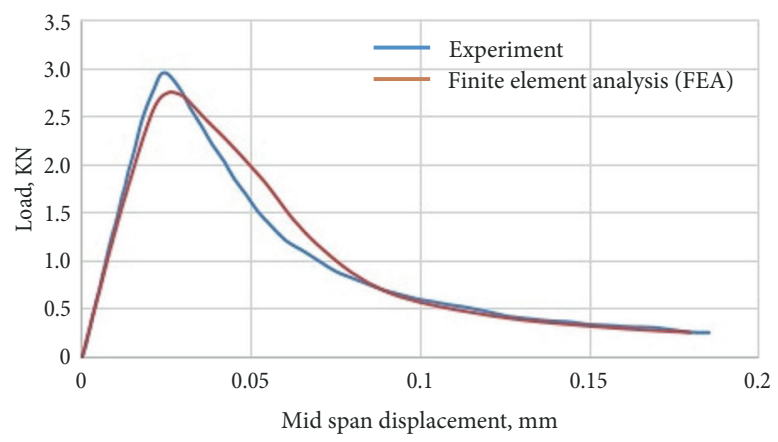

Fig. 22. Comparison of load-displacement responses obtained from FEA and experiment (Le Bellego et al. 2003) in three-point bending notched beam with small size 


\section{Assessment of size effect on the input parameters}

Another important issue in the present paper is to investigate the assessment of the size effect on the input parameters' performance of the CDP model. To this end, three simply supported notched beams with a constant value of tensile strength $f_{t}$ were selected. The geometry of the beams was earlier shown in Figure 18. Parameter calibration was done before for this beam with the size of $h^{\star} L t=8 \mathrm{~cm} * 32 \mathrm{~cm}$ (called small size beam) and in this stage of the study, those optimized parameters outlined in table 2 were used for simulation of two other beams with different sizes entitled as medium and large size beams as follow to investigate whether such input parameters can predict the experimental results or some modifications are needed.

\subsection{Medium size beam}

Medium size beam with dimensions of $h^{\star} L t=16 \mathrm{~cm}^{\star}$ $64 \mathrm{~cm}$ was created in the ABAQUS/CAE. In the finite element simulation of medium size beam, all specifications such as boundary condition, loading, material, mesh type and mesh size were considered similar to the small size beam. In fact, the purpose of using the same characteristics was to investigate only the effects of the size in the results. The load-displacement curves from experimental and FEA for medium size beam were compared in Figure 23. It can be seen from this figure that there is a good agreement between these two types of results. In addition, the load capacity of the beam obtained from FEA is very close to the corresponding quantity obtained from experiment. The percentage of error in computing of peak load from FE analysis is about $1.26 \%$ when it is compared with its corresponding value obtained from test. The observable difference between the softening branches in Figure 23 is due to the fact that for tension stiffening

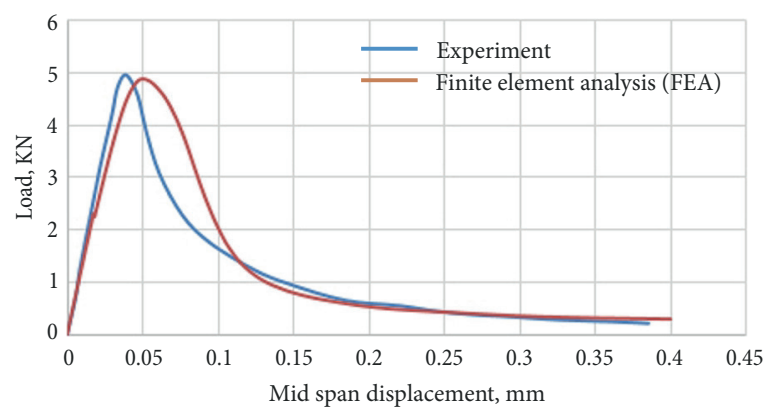

Fig. 23. Comparison load-displacement response: experimental (Le Bellego et al. 2003) and finite element analysis (FEA) in three-point notch beam with medium size of the concrete there are no deterministic experimental observations and only there are some limited analytical relations that using of each of them can lead to different results in softening part of the curve.

\subsection{Large size beam}

The last beam that has been simulated by the ABAQUS/ CAE for studying the effect of specimen size on the input plasticity parameters' performance of the CDP in this research is a large size beam with the dimensions of $h^{\star} L t=32 \mathrm{~cm}{ }^{\star} 128 \mathrm{~cm}$. Similar to the medium size beam, in the finite element simulation of the large size beam, all specifications were considered as the same as to small size beam. The contour of tensile damage resulted from FEA was drawn in Figure 24. As shown in the Figure 24, the critical path for tensile damage is located in the middle of the beam and the elements in this path have been fully damaged like two other beams. The load-displacement curves from experiment (Le Bellego et al. 2003) and FEA for the large size beam were compared in Figure 25. It can be observed in this figure that the results of numerical simulation and experiment have a good coincidence. The percentage of error is about $8.95 \%$. Again, similar to Figure 28, the difference between the softening branches in Figure 30 is due to the lake of valid experimental data for tension stiffening of the concrete in literature.

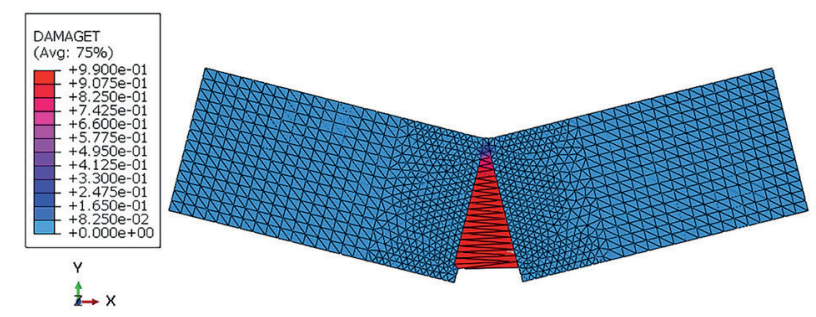

Fig. 24. Tensile damage in three-point notch beam with large size

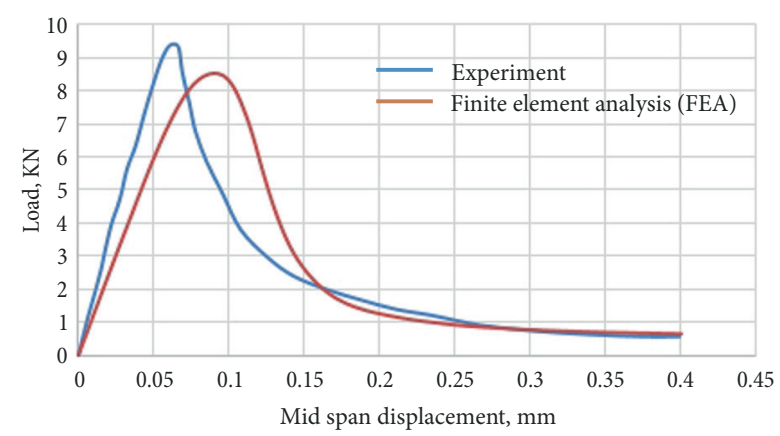

Fig. 25. Comparison load-displacement response: experimental (Le Bellego et al. 2003) and finite element analysis (FEA) in three-point notch beam with large size 
Furthermore, the load capacity of the beam obtained from numerical is very close to that obtained from experiment. By reviewing the results obtained in sections 5.1 and 5.2 it was realized that the optimized input parameters which obtained for the small size beam can simulate very well the behavior of the other different size beams without need for modification. It shows that optimized parameters based on the stress path can take into account the effects of specimen size if the state of stress does not change.

\section{Effect of the shape of the specimen on the calibrated parameters}

The last issue which has been studied in this article is to find the optimum input parameters for the two specimens with a same stress path and different shapes. To this end, a notched disk with a mode I of the tensile cracking similar to the three-point bending beam was chosen. All geometrical details of the disk were shown in the Figure 26 and summarized in the Table 4.

$$
\begin{aligned}
\Pi_{c s} & : \text { Specimen diameter } \\
\Pi_{s b} & \text { : pulling bar diameter } \\
W & : \text { distance from the load axis to the back side } \\
& \text { of the specimen } \\
a & : \text { notch length measured from load axis } \\
b & : \text { specimen thickness } \\
e & : \text { notch width } \\
A_{\text {lig }} & : \text { ligament area }
\end{aligned}
$$

The experiment was carried out by A. Fernández-Canteli et al. (2014). As shown in the Figure 27, the tensile load is applied vertically to the specimen through the steel bars embedded into the specimen. The lower bar is fixed and the upper one is upward ex-

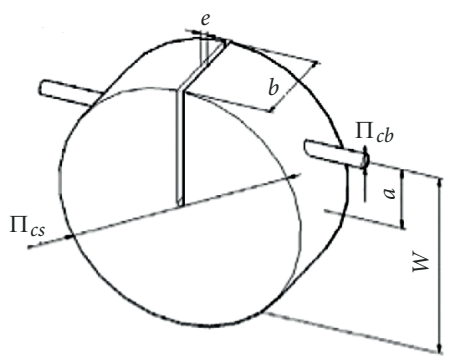

Fig. 26. Characteristic specimen's dimension tended by a hydraulic jack. The compressive and tensile strengths of the concrete are $37 \mathrm{MPa}$ and $2.665 \mathrm{MPa}$ respectively. The finite element model of the disk simulated in ABAQUS software is illustrated in Figure 28. The steel bars were modeled as rigid bodies and a tie constraint between the concrete disk and the steel bars was used. Furthermore, for the concrete specimen, the linear tetrahedral element (C3D4) was used and the size of the elements in the middle part of the disk was considered as $2 \mathrm{~mm}$ and at the rest of the specimen was taken as $4 \mathrm{~mm}$.

The load versus crack opening displacement (COD) curve obtained from ABAQUS model was compared in Figure 29 with the corresponding curve extracted from experiment (Fernández-Canteli et al. 2014). As it can be observed that there is a good agreement between these two types of the results. The percentage of error between peak load of FEA and experiment is about $5.68 \%$.

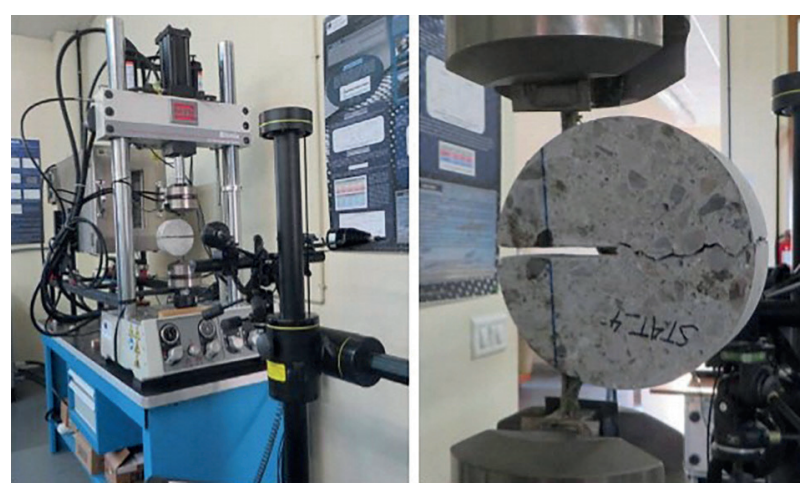

Fig. 27. Experiment setup

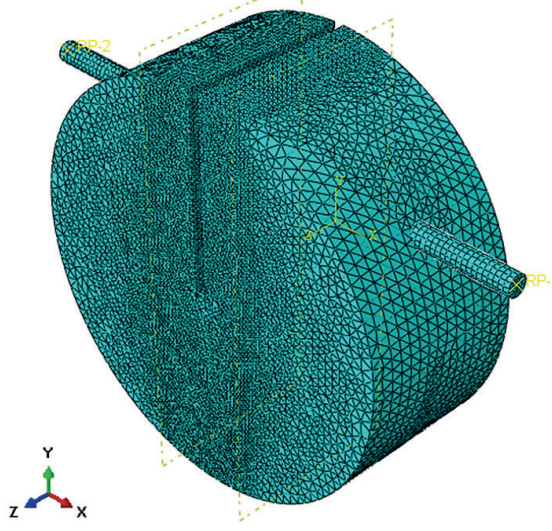

Fig. 28. Finite element (FE) model

Table 4. Dimention of the specimen in $\mathrm{mm}$

\begin{tabular}{|c|c|c|c|c|c|c|}
\hline$\Pi_{c s}$ & $\Pi_{s b}$ & $W$ & $a$ & $b$ & $e$ & $A_{\text {lig }}$ \\
\hline 153 & 8 & 116.4 & 40.2 & 63.5 & 4 & 4838.70 \\
\hline
\end{tabular}




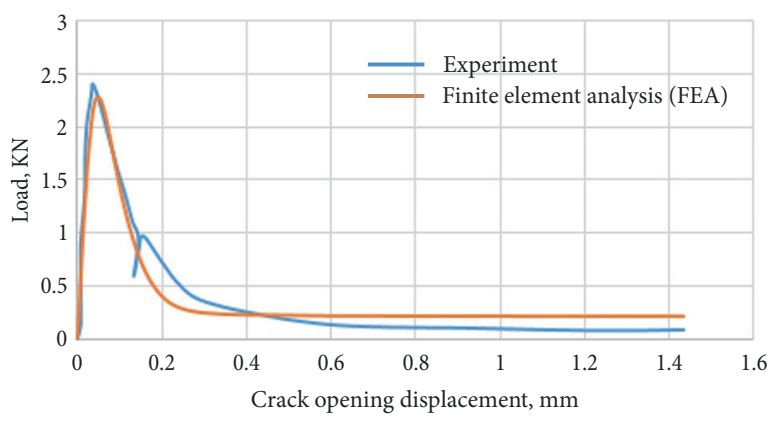

Fig. 29. Load-COD responses: experimental (FernándezCanteli et al. 2014) and finite element analysis (FEA)

One of the relevant parameters that characterize the concrete fracture is its fracture energy. The area under the load-COD is the work of fracture $\left(W_{f}\right)$ and the fracture energy $\left(G_{f}\right)$ is calculated by the following equation:

$$
G_{f}=\frac{W_{f}}{A_{\text {lig }}},
$$

where $A_{\text {lig }}$ is the ligament area. A comparison between the calculated values of the fracture work $\left(W_{f}\right)$ and fracture energy $\left(G_{f}\right)$ obtained in the current study and those obtained from experiment is summarized in table 5 .

Table 5. Summary of the numerical and test results

\begin{tabular}{|c|c|c|c|}
\hline & $W_{f}(J)$ & $G_{f}(J / m)$ & Error (\%) \\
\hline $\begin{array}{c}\text { Experiment } \\
{[17]}\end{array}$ & 475.4 & 98.3 & \multirow{2}{*}{9} \\
\cline { 1 - 2 } $\begin{array}{c}\text { Numerical } \\
\text { simulation }\end{array}$ & 520 & 107.4 & \\
\hline
\end{tabular}

\section{Conclusions}

According to the aforementioned arguments, the main aim of this paper was to investigate the effects of the stress path, specimen size and specimen shape on the optimization of the plastic input parameters of the concrete damage plasticity (CDP) model in the ABAQUS software. For assessing the effect of stress path, three different standard tests: uniaxial compression test, biaxial compression test and three-point bending notched beam test were selected and simulated by the ABAQUS software. The concrete characteristics of the laboratory specimens were very close to each other and this provided a condition to considering just the effect of the stress path on parameters. After examining the results, it was revealed that the stress path can influence on the optimized values of the dilation angle, eccentricity and viscosity parameter. Therefore, it seems beneficial that before starting of each finite element analysis of concrete structures with the CDP model of the ABAQUS software, the optimum values of the above mentioned input parameters are obtained. Other concern of this paper was the assessment of the size effect on the performance of the input parameters of the CDP model. To this end, three-point bending notched beams with three different sizes and constant tensile strength were taken into account and simulated by the ABAQUS software. At first, the input parameters were calibrated for the small size beam and these obtained parameters were used for FE modeling of the medium and large size beams. The obtained results confirmed that those input parameters can simulate very well the behavior of the medium and large size beams. Finally, the last issue that considered in this study is to inquire the effect of the specimen shape on the calibrated parameters of the CDP model. For this purpose, a notched disk with cracking mode I similar to the three-point bending beam was selected and modeled in ABAQUS software and calibrated parameters from three-point bending beam with small size were used in the FE modeling of the disk. The obtained results showed that those input parameters can simulate the behavior of the disk very well. Since, it can be concluded that the studied plastic input parameters of the CDP model in this paper, i.e., dilation angle, eccentricity and viscosity parameter are independent from the effects of the specimen sizes and specimen shape if the state of stress or loading path remains unchanged.

\section{References}

Birtel, V.; Mark, P. 2006. Parameterized finite element modelling of RC beam shear failure, in ABAQUS Users' Conference, May 23-25, 2006, at The Charles Hotel, Cambridge, Massachusetts, USA.

Dassault Systèmes. 2008. Simulia Corp. ABAQUS/CAE Documentation [online], [cited 05 March 2017]. Version 6.8-3. Providence, RI, USA. Available from Internet: www.simulia.com

Fernández-Canteli, A.; Castañón, L.; Nieto, B.; Lozano, M.; Holušová, T.; Seitl, S. 2014. Determining fracture energy parameters of concrete from the modified compact tension test, Frattura ed Integrità Strutturale 30(2014): 383-393.

Kmiecik, P.; Kaminski, M. 2011. Modelling of reinforced concrete structures and composite structures with concrete strength degradation taken into consideration, Archives of Civil and Mechanical Engineering 6: 3. https://doi.org/10.1016/S1644-9665(12)60105-8

Kupfer, H. B.; Gerstle, K. H. 1973. Behaviour of concrete under biaxial stresses, Journal of Engineering Mechanics Division 99(EM4): 853-866. 
Labibzadeh, M. 2015. The numerical simulations of the strengthened RC slabs with CFRPs using standard CDP material model of Abaqus code, European Journal of Environmental and Civil Engineering 19(10): 1268-1287. https://doi.org/10.1080/19648189.2015.1013637

Labibzadeh, M.; Elahifar, T. 2015. An enhanced finite element model for reinforced concrete two-way slabs strengthened with carbon fiber reinforced polymers, Structural Engineering International Journal 1: 81-90. https://doi.org/10.2749/101686614X14043795570093

Labibzadeh, M.; Zakeri, I.; Shoeib, A. 2017a. A new method for CDP input parameter optimization of the ABAQUS software guaranteeing the uniqueness and precision, International Journal of Structural Integrity 8(2): 264-284. https://doi.org/10.1108/IJSI-03-2016-0010

Labibzadeh, M.; Firouzi, A.; Ghafouri, H. R. 2017b. Structural performance evaluation of an aged structure using a modified plasticity model in inverse solution method. Article in Press.

Le Bellego, C.; Dube, J. F.; Pijaudier-Cabot, G.; Gerard, B. 2003. Calibration of nonlocal damage model from size effect tests, European Journal of Mechanics A/Solids 22: 33-46. https://doi.org/10.1016/S0997-7538(02)01255-X

Lee, J.; Fenves, G. L. 1998. Plastic-damage model for cyclic loading of concrete structures, Journal of Engineering Mechanics 124(8): 892-900. https://doi.org/10.1061/(ASCE)0733-9399(1998)124:8(892)

Lubliner, J.; Oliver, J.; Oller, S.; Onate, E. 1989. Plastic-damage model for concrete, International Journal of Solids Structures 25(3): 299-326. https://doi.org/10.1016/0020-7683(89)90050-4

Massicotte, B.; Elwi, A. E.; MacGregor, J. G. 1990. Tensionstiffening model for planar reinforced concrete members, Journal of Structural Engineering, ASCE 116(1): 3039-3058. https://doi.org/10.1061/(ASCE)0733-9445(1990)116:11(3039)
Popovics, S. 1973. A numerical approach to the complete stress strain curve for concrete, Cement ConcrRes 3(5): 583-599. https://doi.org/10.1016/0008-8846(73)90096-3

Rodrigues, J.; Martinez, F.; Marti, J. 2013. Concrete constitutive model, calibration and application, in Simulia community conference, 21-24 May, Vienna, Austria.

Sadeghi, J. M.; Fathali, M. 2007. Deterioration analysis of concrete bridges under inadmissible loads from the fatigue point of view, Sciatica Iranica 14(3): 185-192.

Sadeghi, J. M.; Fathali, M. 2012. Grillage analogy applications in analysis of bridge decks, Australian Journal of Civil Engineering 10(1): 23-36. https://doi.org/10.7158/C10-670.2012.10.1

Sadeghi, J.; Liravi, H.; Esmaeili, H. 2017. Experimental investigation on loading pattern of railway concrete slabs, Construction and Building Materials 153(30): 481-495. https://doi.org/10.1016/j.conbuildmat.2017.07.025

Shima, H.; Chou, L.; Okamura, H. 1987. Micro and macro models for bond behavior in reinforced concrete, Journal of the Faculty Engineering, University of Tokyo 39(564): 297-316.

Sümer, Y.; Aktaş, M. 2015. Defining parameters for concrete damage plasticity model, Challenge Journal of Structural Mechanics 1(3): 149-155.

Szczecina, M, S.; Winnicki, A. 2015. Calibration of the CDP model parameters in ABAQUS, in the 2015 World Congress on advances in structural engineering and mechanics (ASEM15), August 25-29, Incheon, Korea.

Zi, G.; Rabezuk, T.; Wall, W. 2007. Extended meshfree methods without branch enrichment for cohesive cracks, Computational Mechanics 40: 367-382. https://doi.org/10.1007/s00466-006-0115-0

Wang, T.; Hus, T. T. C. 2001. Nonlinear finite element analysis of concrete structures using new constitutive models, ComputStruct 79(32): 2781-2791. https://doi.org/10.1016/S0045-7949(01)00157-2

Mojtaba LABIBZADEH, the corresponding author, is born in 1976 in Ahvaz at Khouzestan province of Iran. He received his B. S. in civil engineering at 1998, his M. Sc in hydraulic structural engineering at 2000 and his PhD in structural engineering at 2006. $\mathrm{He}$ is a faculty member of department of civil engineering of faculty of engineering of Shahid Chamran university of Ahvaz since 2006. He published several papers in the field of simulation of concrete structures.

Reza HAMIDI is the M.Sc student of structural engineering of department of civil Engineering, faculty of engineering, Shahid Chamran university of Ahvaz, Iran. 